\title{
pH Sensing of Printed Flexible Sensors
}

\author{
Anindya Nag, Omer Faruk Dorsum, Subhas Mukhopadhyay \\ School of Engineering, Macquarie University \\ Sydney, Australia \\ Email: anindya1991@gmail.com
}

\author{
Jurgen Kosel \\ Computer Electrical and Mathematical Sciences and \\ Engineering Division \\ King Abdullah University of Science and Technology, \\ Thuwal, Saudi Arabia
}

\begin{abstract}
This paper presents the fabrication and implementation of some of the printed flexible sensors develop with laser cutting technique. Different types of elastomeric and conductive materials were used to fabricate the electrodes and substrates of the sensor prototypes. The developed prototypes were then used as $\mathrm{pH}$ sensors to determine their behavior over a range of $\mathrm{pH}$ values at the acidic and basic spectrum. The behavior of these prototypes proved their potential to be used for electrochemical sensing in different health and environmental applications.
\end{abstract}

\section{Keywords-CNTs; PDMS; Graphene; Polyimide; PET; pH.}

\section{INTRODUCTION}

The use of sensors in temperature [1], humidity [2] and $\mathrm{pH}$ [3] has been done for quite some time. The advantages of using sensors for these applications lies in easy and quick monitoring of the ambiance conditions where it is difficult for a person to reach. Earlier, during the use of silicon sensors for these applications [4-6], certain advantages like quick response, high sensitivity and durability of the sensors were highlighted. But certain disadvantages like high cost of fabrication, high input power and minimized chances to be operated at extreme conditions led the researchers to opt for alternative options. Since the last decade, flexible sensors [7] has become a very popular choice for a dynamic range of applications due to their improved electrical, mechanical and thermal characteristics. Researchers are constantly trying to develop sensor prototypes with reduced cost of fabrication, increased functionality, higher sensitivity and durability. Different types of elastomers like Polydimethylsiloxane (PDMS) [8], Polyethylene terephthalate (PET) [9], Polyimide (PI) [10], etc. has been used as elastomers to develop the substrates of the sensor prototypes. Similarly, a variety of conductive materials like Carbon Nanotubes (CNTs) [11], graphene [12], gold [13], etc. has been opted to develop the electrodes of the sensor prototypes. The type of the processing materials depends on primarily on the fabrication technique and application of the sensors. Among the fabrication techniques, laser cutting technique [14] has been one of the options can be favored over the other fabrication techniques like screen printing [15], photolithography [16] and contact printing [17] due to their low cost and easy sample preparation, development of very flexible devices and large-scale production in a short time. This paper depicts the design and development of three flexible sensor prototypes that have been developed with different elastomers and conductive materials.
The sensors have been used for $\mathrm{pH}$ sensing at the acidic and basic spectrum to portray their potentiality to be used as $\mathrm{pH}$ sensors in different applications.

\section{FABRICATION OF THE SENSORS}

The fabrication of the sensor patches was done at fixed temperature $\left(25^{\circ} \mathrm{C}\right)$ and humidity $(50 \% \mathrm{RH})$ conditions. The first sensor prototype was based on the use of CNTs and PDMS as the electrode and substrate respectively to develop the sensor patches. The difference in the fabrication process of types of sensor patches from that of the other ones lied in the use of a nanocomposite (NC) layer to develop the electrodes. Figure 1 shows the schematic diagram of the fabrication process. PDMS (SYLGARD $^{\circledR} 184$, Silicon Elastomer Base) was formed by mixing the base elastomer (pre-polymer) and curing agent (cross-linking agent) at a ratio of 10:1 respectively and cast on a Poly (methyl methacrylate) (PMMA) template. The reason PDMS was chosen as a substrate was due to its low cost, compatible nature and hydrophobicity. Proper adherence and non-reactive nature to the cured PDMS are the two reasons due to which PMMA was considered as the platform to form the sensor patches. The height of the cast PDMS was adjusted to around 1000 microns by a casting knife (SHEEN, $1117 / 1000 \mathrm{~mm})$ to define the thickness of the sensor patches. Followed by the desiccation of the sample for an hour to remove the air bubbles, it was cured in the oven at $80^{\circ} \mathrm{C}$ for two hours.

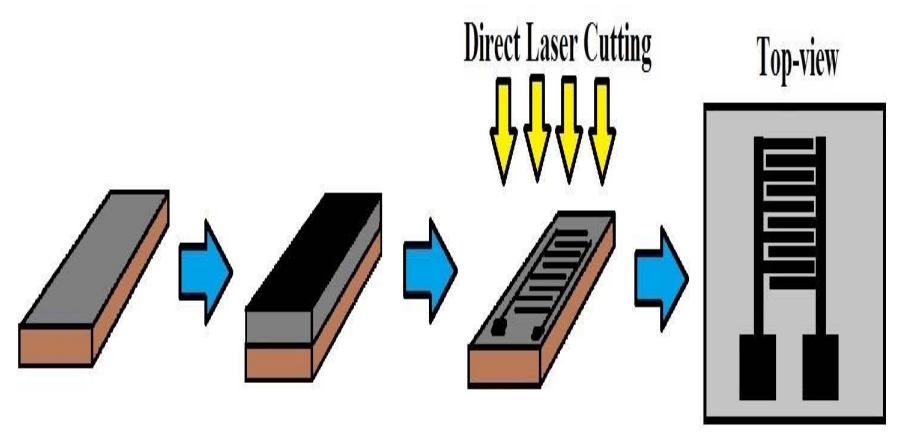

Figure 1: Schematic diagram of fabrication steps of the CNT-PDMS sensor patches.

A NC was formed by mixing carboxylic group (-COOH) functionalized Multi-Walled Carbon nanotubes (MWCNTs) 
(Aldrich, 773840-100G) with PDMS at definite proportions to form the conductive part of the sensor patches. Functionalized MWCNTs was chosen as conductive material due to their high electrical conductivity, mechanical flexibility and increased ability to form nanocomposites with elastomers due to high interfacial bonds with the polymers. Different proportions of MWCNTs in wt. \% was mixed and tested in terms of flexibility and conductivity to determine the optimized value. Finally, 4 wt. \% of MWCNTs was fixed to mix in PDMS to form the nanocomposites for the sensor patches. Figure 2 shows the SEM image of the optimized MWCNTs and PDMS values. The black regions shown in the image are the MCNTs while their counterparts are the PDMS. The NC layer was cast on top of the cured PDMS whose height was also adjusted to around 600 microns.

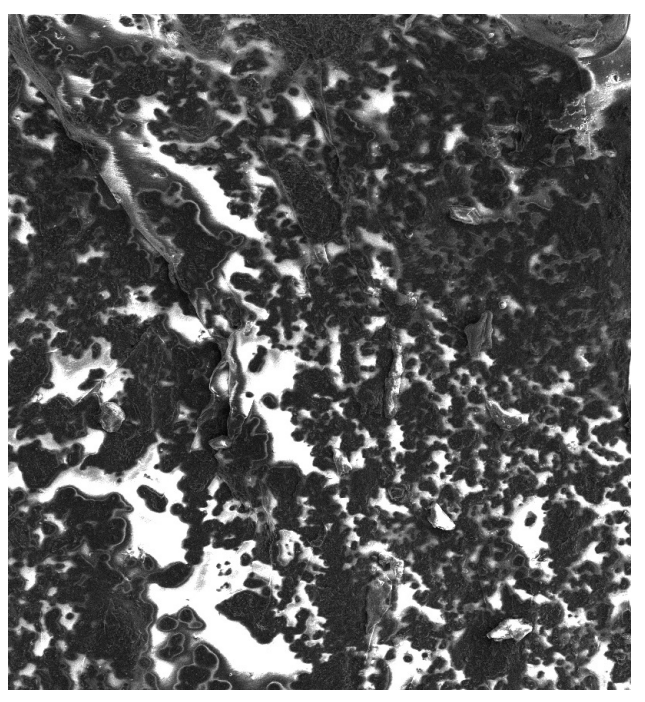

Figure 2: SEM image of the optimized CNT wt. \% in the nanocomposite.

The samples were again desiccated and cured at the same time duration as before to complete the solidifying process. Finally, the sample was taken for laser-cutting where the selective parts of the NC layer was scanned off to form the electrodes of the sensor patches. Universal Laser System (Model: OLS 6.75 $\mathrm{CO}_{2}$ laser system, laser spot diameter: 150 microns) was used for the laser-cutting purposes where the designs of the electrodes were drawn on the designing software (CREO Parametric 2.0) associated with it. Power $(\mathrm{W})$, speed $(\mathrm{m} / \mathrm{min})$, and $\mathrm{z}$-axis $(\mathrm{mm})$ are the three parameters that were optimized in the laser-cutting process. Power and speed defined the intensity of the laser cutting process and the movement of the nozzle along $\mathrm{x}$ and $\mathrm{y}$ directions over the sample respectively, whereas z-axis defined the focal point of the laser beam on the sample. The optimized values of the laser parameters were $24 \mathrm{~W}, 70 \mathrm{~m} / \mathrm{min}$ and $1 \mathrm{~mm}$. Inverse scanning technique was operated to form the electrodes by scanning off parts of the NC layer other than electrodes. Figure 3 shows the front and rear view of the developed CNTPDMS sensor patches. Four pairs of interdigitated electrodes were present with a total sensing area of $22.5 \mathrm{~mm}^{2}$. The thickness of each electrode finger was 200 microns with a gap of 100 microns between two consecutive fingers. The sensor patches were characterized and used for monitoring different physiological parameters like limb movements and respiration [18].

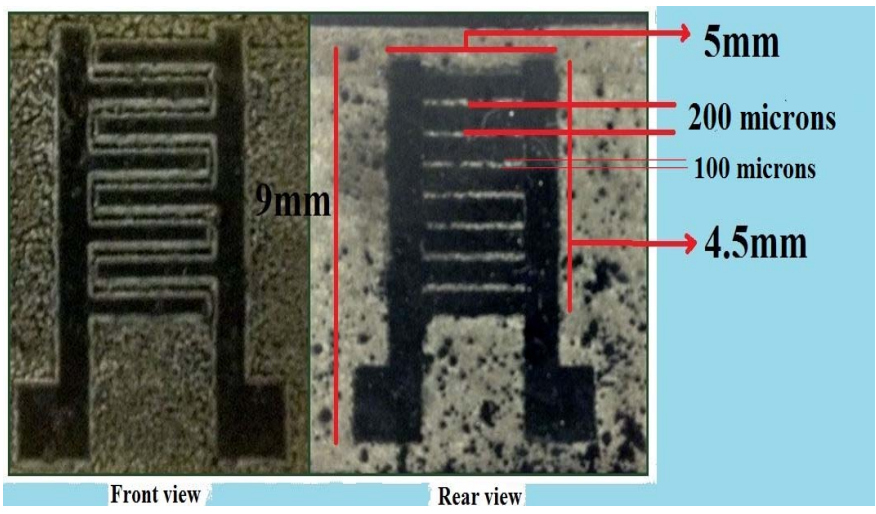

Figure 3: Front and rear view of the developed CNT-PDMS sensor patches.

The second type of sensor patches were developed from metallized commercial films. The difference of these sensor patches from that of the other ones lied on the high flexibility of the sensor patches and the use of a single material to fabricate them. Figure 4 shows the schematic diagram of the fabrication process. Metallized PET films (HO-107) were used as the substrate material whose one side was coated with Aluminum (Al). The thicknesses of the PET and Al layer were 500 microns and 300 microns respectively. The metallized polymer films were attached on a glass substrate with biocompatible tapes $\left(3 \mathrm{M}^{\mathrm{TM}} \mathrm{VHB}^{\mathrm{TM}}\right)$ and taken for laser-ablation on the $\mathrm{Al}$ side.

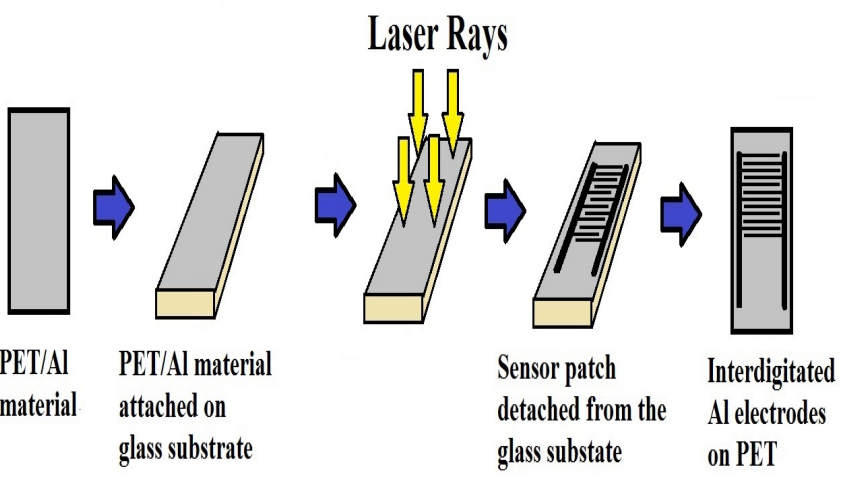

Figure 4: Schematic diagram of the fabrication steps of the Al-PET sensor patches.

The difference between the polymer and the metallic side can be determine by higher electrical conductivity and shinier nature of the later one than the former one. An optimization as done on the laser parameters to determine the thickness of the electrode lines. The final values were $2.6 \mathrm{~W}, 52.5 \mathrm{~m} / \mathrm{min}$ and $1.2 \mathrm{~mm}$ for the power, speed and $\mathrm{z}$-axis respectively to obtain the electrode lines of 41.2 microns. Figure 5 shows the SEM image of the developed electrode lines on the Al side. It is seen even though the lines are smooth and perpendicular to the sensor, there are tiny bumps present on its surface. These bumps were generated as a result of the melting and coagulation of the 
$\mathrm{Al}$ as a result of heat generated by the laser. The fabricated sensor patches were characterized and used for tactile sensing applications [19]. Figure 6 shows the front and rear view of the developed Al-PET sensor patches. The sensor patches had twelve pairs of interdigitated electrode fingers, each having a length and width of $1.2 \mathrm{~mm}$ and 41 microns respectively.

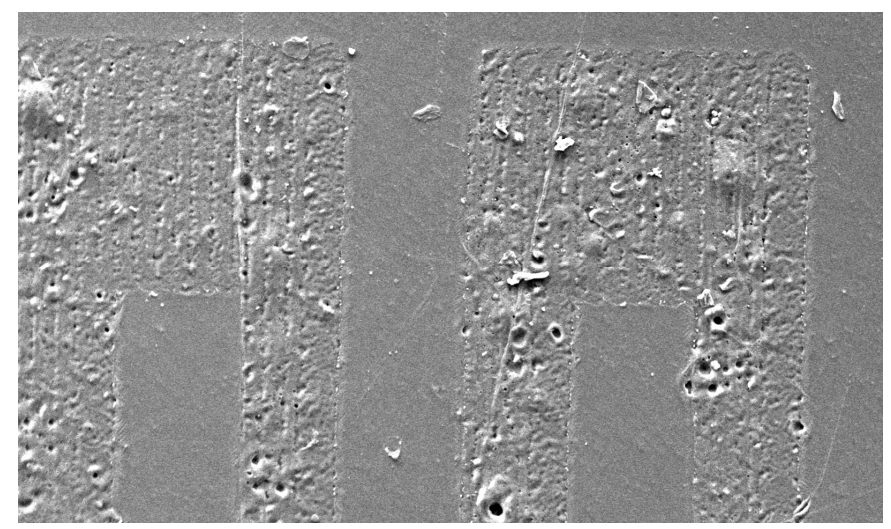

Figure 5: SEM images of the electrodes of the Al-PET sensor patches depicting the laser-cut of the $\mathrm{Al}$ side.

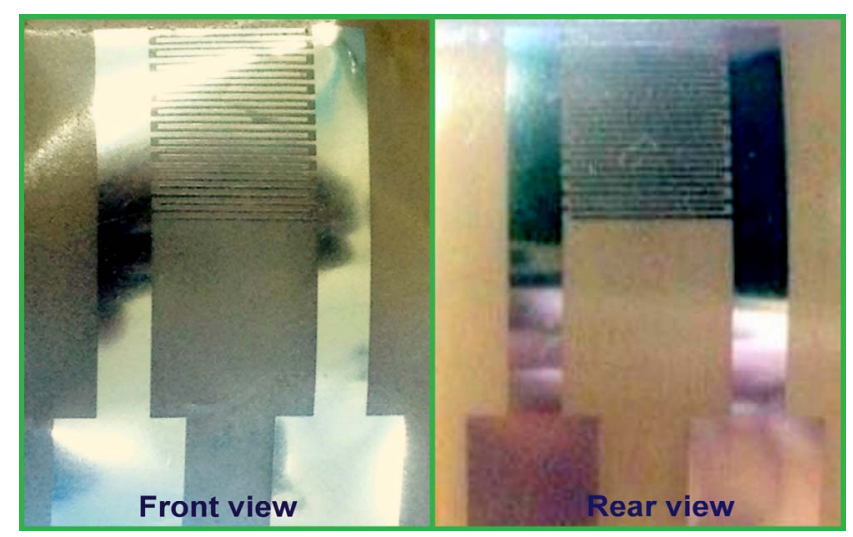

Figure 6: Front and rear view of the Al-PET sensor patches.

The third sensor prototype was developed by laser-induction of commercial PI films. The difference of the fabrication process of these types of sensor patches from that of the other ones lied in the high conductivity of the fabricated sensor patches and generation of conductive material from laser-induction of commercial polymer films. Figure 7 shows the individual steps of the fabrication process. Commercial PI films (Zibo Zhongnan Plastics Co., Ltd.) were taken and attached to fixed platform for laser patterning. Followed by designing the electrodes on the system, laser-induction was done to photothermally convert the $\mathrm{sp}^{3}$ hybridized carbon atoms of the PI films to $\mathrm{sp}^{2}$ hybridized carbon atoms of graphene. Similar to the first and second prototypes, the laser parameters were adjusted and optimized to develop graphene out of the PI films. The power, speed and z-axis values were $9 \mathrm{~W}, 70 \mathrm{~m} / \mathrm{min}$ and $1 \mathrm{~mm}$ respectively.

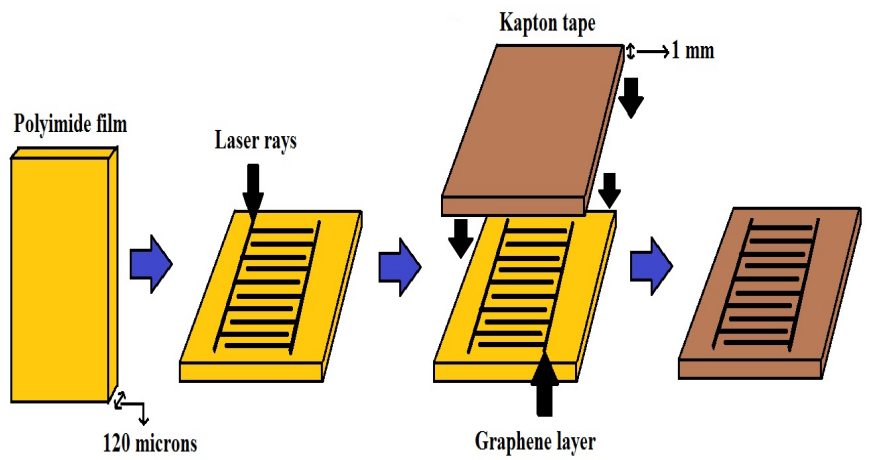

Figure 7: Schematic diagram of fabrication of the graphene-PI sensor patches.

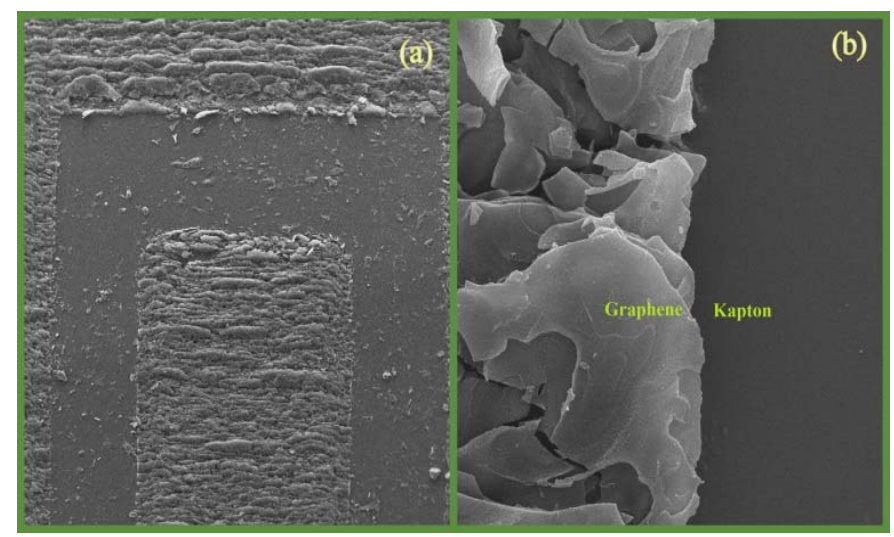

Figure 8: SEM image of the (a) top-view of the electrodes and (b) single electrode line of the transferred graphene powder on the Kapton tapes.

Followed by the generation of the laser-induced graphene, the conductive material was transferred to commercially available Kapton tapes to use them as electrodes in the sensor patches. Even though the substrate and the transferring material was of same type, there are two reasons due to which laser induction was not done directly on the Kapton tapes. Firstly, the sticky nature of the tapes would have tampered the design of the electrodes. Secondly, it would have been difficult to operate the laser-induction process on the Kapton tapes (1000 microns) due to their thickness compared to that of the PI films (120 microns). The transfer of graphene was done manually by applying pressure preceded by the placement of the Kapton tapes over the conductive material. Figures 8(a) and 8(b) show the SEM images of the top-views of the transferred graphene on Kapton tapes. It is seen from the figure that the transfer was clean on the Kapton tapes which nullified the use of any postprocessing step to achieve that. Figure 9 shows the final product that was characterized and used for monitoring different environmental $[20,21]$ applications. The sensor patches had six pairs of interdigitated electrode fingers with an overall sensing area of $96 \mathrm{~mm}^{2}$. The length and width of each electrode finger was 500 microns and 100 microns respectively. 


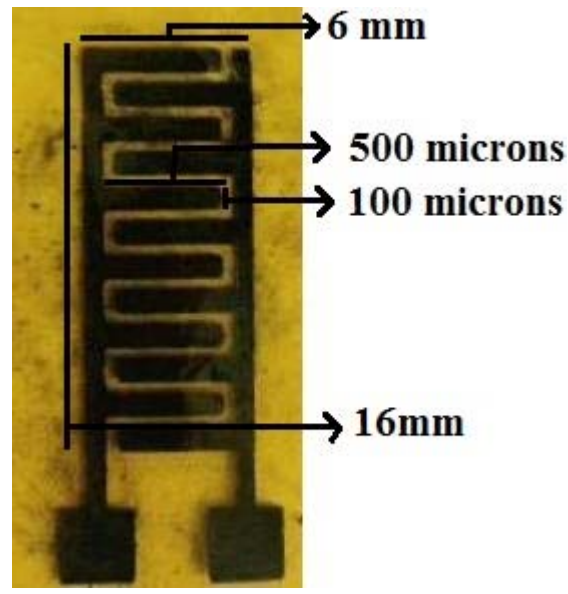

Figure 9: Graphene-PI sensor based on laser-induced process of commercial polyimide films.

\section{WORKING PRINCIPLE OF THE SENSORS}

The operating principle of the sensor patches replied on the structure of the electrodes of the three sensor prototypes. Due to their interdigitated structure, the sensor patches operated on capacitive principle [22]. The advantages of these type of sensor patches lied on the non-invasive, single-sided measurement of different dielectric materials $[23,24]$. When a potential difference would be applied on the two electrodes, the generated electric field would bulge from one electrode to another of opposite polarity due to its planar nature.
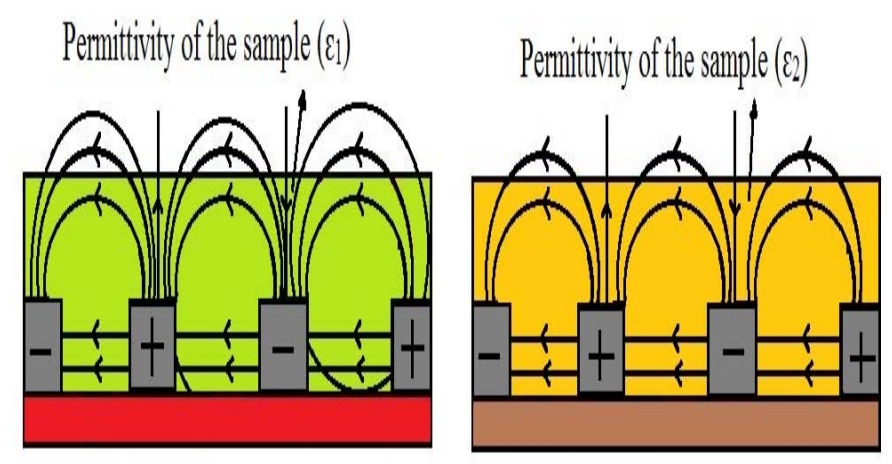

Figure 10: Schematic diagram of the operating principle of the developed sensor prototypes.

If any material is kept in proximity or in contact with the electrodes, the characteristics of the electric field would as change as it penetrates through the material. The change in the characteristics would result due to the change in the relative permittivity $\left(\varepsilon_{\mathrm{r}}\right)$, which changes the overall response of the sensor patches. This change in characteristics would be studied to determine the attributes of the material. Interdigitated sensors have been largely used for monitoring purposes in different health [25, 26], environmental [27, 28] and industrial [29, 30] applications.

\section{EXPERIMENTAL RESULTS}

Figure 11 shows the schematic diagram of the setup followed during the experimental process. The sensor patches were attached with the biocompatible tapes $\left(3 \mathrm{M}^{\mathrm{TM}} \mathrm{VHB}^{\mathrm{TM}}\right)$ to a wire board to restrict their movements under water during measurements. The bonding pads of the sensor patches were connected to an impedance analyzer (HIOKI IM 3536) via Kelvin probes to determine the changes taking place with respect to the different $\mathrm{pH}$ solutions. The response was taken in terms of the change in phase angle $(\Phi)$ with respect to frequency. A voltage of $1 \mathrm{~V}$ was given as the input to the impedance analyzer while the frequency range was swept between $10 \mathrm{~Hz}$ and 100 $\mathrm{kHz}$. The responses shown here are only concerned with the operating frequency regions for the different $\mathrm{pH}$ values. The impedance analyzer was connected to a computer with a USB cable to collect the data on Microsoft Excel via automatic data acquisition algorithm. Five acidic (1.71, 2.75, 3.47, 4.27 and 5.35) were made and four basic $(8.5,9.41,10.49$ and 11.59) were made to perform the experiments. The acidic and basic solutions were made by mixing Sulfuric acid $\left(\mathrm{H}_{2} \mathrm{SO}_{4}\right)$ and Sodium Hydroxide $(\mathrm{NaOH})$ solutions respectively to de-ionized water (pH: 6.71, resistance: $18.2 \mathrm{M} \Omega$ ). The testing was done three times and an average was taken to ensure the repeatability of the responses.

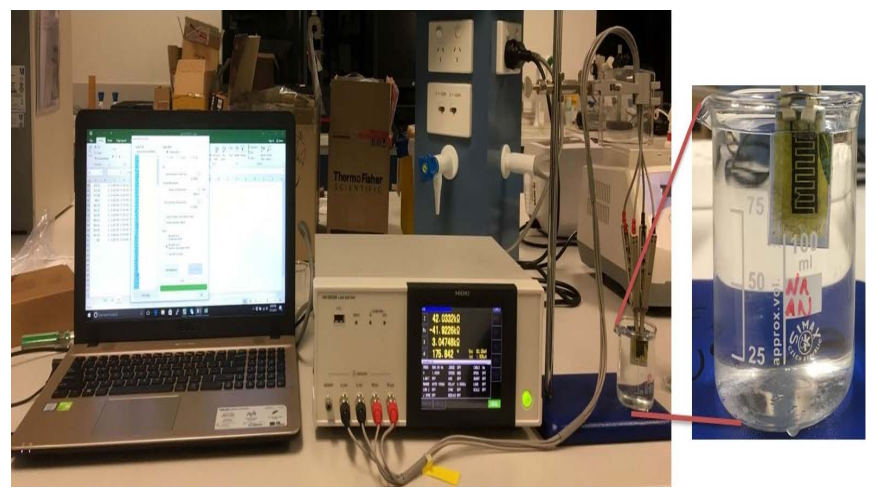

Figure 11: Experimental setup depicting the connection of the sensor patches to the impedance analyzer for monitoring the response to different $\mathrm{pH}$ values.

Figures 12-14 show the response of the sensor patches towards the acidic and basic spectrum. It is seen from the response of the CNT-PDMS sensor patches (Figure 12) that the optimum frequency region for the entire $\mathrm{pH}$ range is high. This might be due to the nature of the sensor patches where their formation was based on two separate layers which effects the resistive part of the sensor. It is seen that the behavior of the Al-PET sensor patches (Figure 13) towards the $\mathrm{pH}$ values that even though they responded to lower frequency regions, the responses were not as smooth as that of the CNT-PDMS ones. This might be due to the coagulative nature of the electrodes lines which took place during the fabrication process. Although due to the lower thickness of the electrodes and substrates of the Al-PET sensor patches, the electrochemical reactive happened at lower frequencies, the uneven sensing surface of the electrodes also effected the resistive nature of the sensor patches. It is also seen that the nature of change of response of Al-PET sensor patches are different to that of CNT-PDMS and graphene-PI sensor patches. The increase of $\mathrm{pH}$ values with respect to frequency can be attributed to the formation of the sensors using a singular material. 


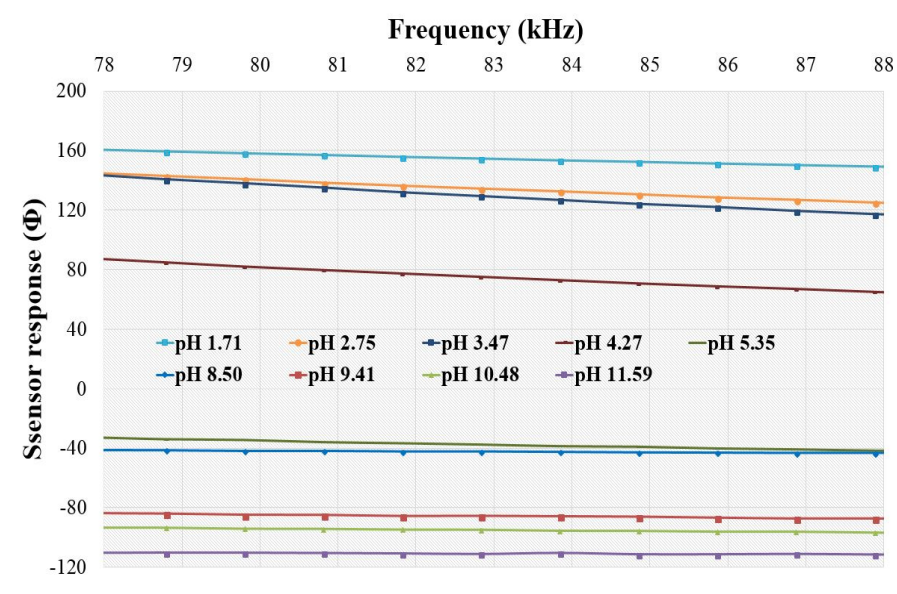

Figure 12: Response of the CNT-PDMS sensor patches towards the acidic and basic $\mathrm{pH}$ values.

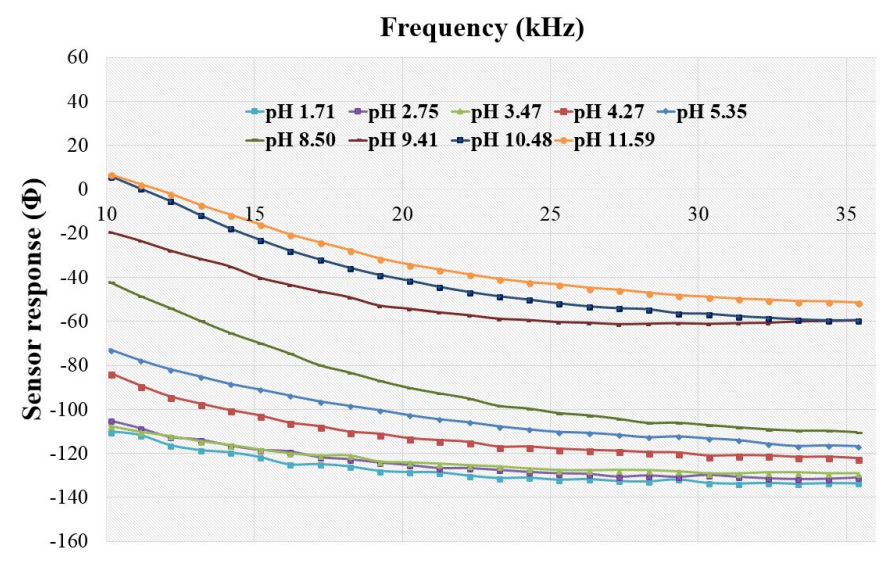

Figure 13: Response of the Al-PET sensor patches towards the acidic and basic $\mathrm{pH}$ values.

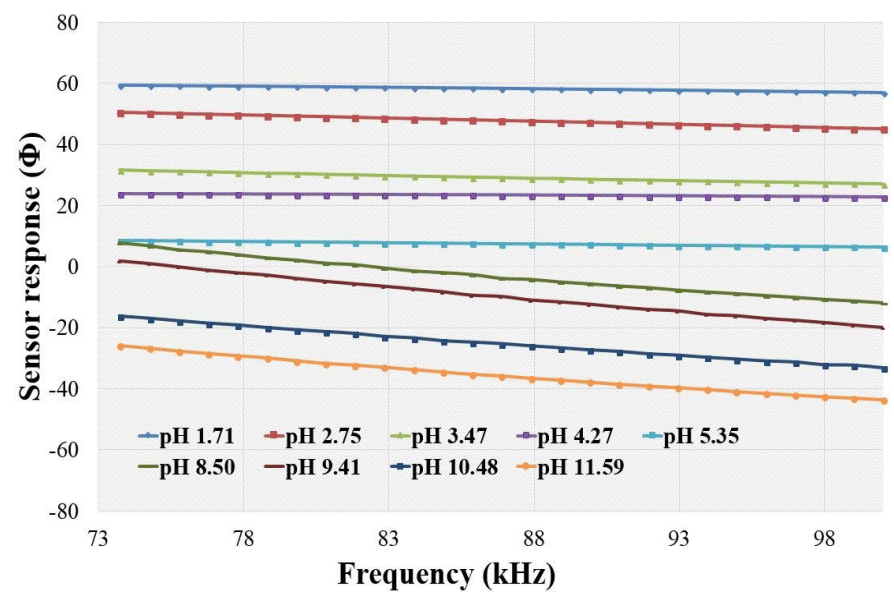

Figure 14: Response of the graphene-PI sensor patches towards the acidic and basic $\mathrm{pH}$ values.

The responses of the graphene-PI sensor patches (Figure 14) was the best towards the $\mathrm{pH}$ values for the three types of fabricated sensors. It is seen that the differences in the sensor response changed symmetrically with respect to each $\mathrm{pH}$ value.
The reason for this optimum response can be attributed to the porous nature and high conductivity of the electrodes. The operating region for the graphene-PI sensor patches was like that of the CNT-PDMS ones due to the inclusion of two separate layers of electrodes and substrates during their fabrication processes.

\section{CONCLUSION}

The paper presents the fabrication and implementation of three different types of flexible printed sensors. The differences lied in the different ways of fabrication and type of processing material related to them. The developed sensor patches were then experimented with $\mathrm{pH}$ values in acidic and basic spectrum. The analysis was done using an impedance analyzer to determine the change in phase angle $(\Phi)$ with respect to frequency for different $\mathrm{pH}$ values. The maximum differences in the response was found out for the graphene-PI sensor patches for both the acidic and basic medium. Apart from their significant response towards $\mathrm{pH}$ sensing, other advantages of these sensor patches lied in their low fabrication cost and easy operationality. These sensor patches have a high potential to be used in combination with other impedimetric sensors for developing a complete sensing system.

\section{ACKNOWLEDGEMENT}

The authors are thankful to King Abdullah University of Science and Technology (KAUST), Saudi Arabia, to provide the research facilities to fabricate the sensor patches. They would also thank the Macquarie University, Australia for providing the research conditions to perform experiments with the developed sensor patches.

\section{REFERENCES}

[1] Y. Moser and M. A. Gijs, "Miniaturized flexible temperature sensor," Journal of microelectromechanical systems, vol. 16, pp. 1349-1354, 2007.

[2] P.-G. Su and C.-S. Wang, "Novel flexible resistive-type humidity sensor," Sensors and Actuators B: Chemical, vol. 123, pp. 10711076, 2007.

[3] N. Ferrer-Anglada, et al., "Transparent and flexible carbon nanotube/polypyrrole and carbon nanotube/polyaniline pH sensors," physica status solidi (b), vol. 243, pp. 3519-3523, 2006.

[4] M. J. Schöning, et al., "A highly long-term stable silicon-based pH sensor fabricated by pulsed laser deposition technique," Sensors and Actuators B: Chemical, vol. 35, pp. 228-233, 1996.

[5] Z. Rittersma, et al., "A novel surface-micromachined capacitive porous silicon humidity sensor," Sensors and Actuators B: Chemical, vol. 68, pp. 210-217, 2000.

[6] K. S. Szajda, et al., "A low noise, high resolution silicon temperature sensor," IEEE Journal of Solid-State Circuits, vol. 31, pp. 13081313, 1996.

[7] S. Stassi, et al., "Flexible tactile sensing based on piezoresistive composites: A review," Sensors, vol. 14, pp. 5296-5332, 2014. T. H. da Costa and J.-W. Choi, "A flexible two dimensional force sensor using PDMS nanocomposite," Microelectronic Engineering, vol. 174, pp. 64-69, 2017.

[9] U. Yaqoob, et al., "Highly flexible room temperature NO 2 sensor based on MWCNTs-WO 3 nanoparticles hybrid on a PET substrate," Sensors and Actuators B: Chemical, vol. 221, pp. 760768, 2015 .

[10] Y. Qin, et al., "Lightweight, superelastic, and mechanically flexible graphene/polyimide nanocomposite foam for strain sensor application," ACS nano, vol. 9, pp. 8933-8941, 2015. 
[11] X. Peng, et al., "A flexible humidity sensor based on KC-MWCNTs composites," Applied surface science, vol. 387, pp. 149-154, 2016.

[12] C. Lou, et al., "Flexible Graphene Electrodes for Prolonged Dynamic ECG Monitoring," Sensors, vol. 16, p. 1833, 2016.

[13] C. Tasaltin and F. Basarir, "Preparation of flexible VOC sensor based on carbon nanotubes and gold nanoparticles," Sensors and Actuators B: Chemical, vol. 194, pp. 173-179, 2014.

[14] A. K. Ghavidel, et al., "Effect of carbon nanotubes on laser cutting of multi-walled carbon nanotubes/poly methyl methacrylate nanocomposites," Optics \& Laser Technology, vol. 67, pp. 119-124, 2015.

[15] W.-Y. Chang, et al., "A large area flexible array sensors using screen printing technology," Journal of display technology, vol. 5, pp. 178$183,2009$.

[16] S. Khumpuang, et al., "Photolithography for minimal fab system," IEEJ Transactions on sensors and micromachines, vol. 133, pp. 272-277, 2013.

[17] J. Perelaer, et al., "Printed electronics: the challenges involved in printing devices, interconnects, and contacts based on inorganic materials," Journal of Materials Chemistry, vol. 20, pp. 8446-8453, 2010.

[18] A. Nag, et al., "Flexible carbon nanotube nanocomposite sensor for multiple physiological parameter monitoring," Sensors and Actuators A: Physical, vol. 251, pp. 148-155, 2016.

[19] A. Nag, et al., "Tactile Sensing From Laser-Ablated Metallized PET Films," IEEE Sensors Journal, vol. 17, pp. 7-13, 2016.

[20] A. Nag, et al., "Sensing System for Salinity Testing Using Laserinduced Graphene Sensors," Sensors and Actuators A: Physical, 2017.

[21] A. Nag and S. C. Mukhopadhyay, "Fabrication and implementation of printed sensors for taste sensing applications," Sensors and Actuators A: Physical, vol. 269, pp. 53-61, 2018.

[22] A. Nag, et al., "Influence of temperature and humidity on carbon based printed flexible sensors," in Sensing Technology (ICST), 2017 Eleventh International Conference on, 2017, pp. 1-6.

[23] M. S. A. Rahman, et al., "Novel Planar Interdigital Sensors," in Novel Sensors for Food Inspection: Modelling, Fabrication and Experimentation, ed: Springer, 2014, pp. 11-35.

[24] A. V. Mamishev, et al., "Interdigital sensors and transducers," Proceedings of the IEEE, vol. 92, pp. 808-845, 2004.

[25] A. Nag, et al., "Strain induced graphite/PDMS sensors for biomedical applications," Sensors and Actuators A, vol. 271, pp. 257-269, 2018.

[26] A. Nag, et al., "Urinary incontinence monitoring system using laserinduced graphene sensors," in SENSORS, 2017 IEEE, 2017, pp. 13.

[27] A. Nag, et al., "Novel Sensing Approach for LPG Leakage Detection: Part I-Operating Mechanism and Preliminary Results," IEEE Sensors Journal, vol. 16, pp. 996-1003, 2016.

[28] A. Nag, et al., "Novel Sensing Approach for LPG Leakage Detection-Part II: Effects of Particle Size, Composition, and Coating Layer Thickness," IEEE Sensors Journal, vol. 16, pp. 10881094, 2016.

[29] A. Nag, et al., "Performance Analysis of Flexible Printed Sensors for Robotic Arm Applications," Sensors and Actuators A: Physical, 2018.

[30] A. Nag, et al., "Transparent biocompatible sensor patches for touch sensitive prosthetic limbs," in Sensing Technology (ICST), 2016 10th International Conference on, 2016, pp. 1-6. 